\title{
Phosphate and Oxysterols May Mediate an Inverse Relationship Between Atherosclerosis and Cancer
}

\author{
Authors: \\ Ronald B. Brown \\ School of Public Health and Health Systems, University of Waterloo, \\ Waterloo, Canada \\ Correspondence to r26brown@uwaterloo.ca \\ Disclosure: \\ The author has declared no conflicts of interest. \\ Received: \\ 13.03 .20 \\ Accepted: \\ 22.05 .20 \\ Keywords: \\ Atherosclerosis, cancer, Mediterranean diet, oxysterols, phosphate toxicity. \\ Citation: \\ EMJ Oncol. 2020;8[1]:114-121.
}

\begin{abstract}
The peer-reviewed literature has reported an inverse relationship between atherosclerosis and cancer for almost 100 years, but no causative mechanism has been established to explain this puzzling relationship. More recent research has reported an association between tumourigenesis and phosphate toxicity from dysregulated phosphate metabolism, and an association has also been reported between atherosclerosis and cholesterol oxidation products or oxysterols. The present review article synthesises these research findings and proposes that an inverse relationship between the associated risk of cancer and atherosclerosis may be mediated by tumourigenic and atherogenic dietary patterns containing inverse proportions of dietary phosphate and oxysterols. Low-fat animalbased foods generally have reduced cholesterol and oxysterol levels and relatively higher protein and phosphate levels, and dietary patterns containing these foods are associated with reduced atherosclerosis risk and increased cancer risk. By comparison, full-fat animal-based foods are higher in cholesterol and oxysterols and relatively lower in protein and phosphate, and dietary patterns containing these foods are associated with increased atherosclerosis risk and reduced cancer risk. Fruits, vegetables, and plant-based fats generally have lower phosphate levels and no cholesterol, and dietary patterns associated with increased amounts of these foods, such as the Mediterranean diet, reduce risk for both cancer and cardiovascular disease.
\end{abstract}

\section{INTRODUCTION}

"If it's not one thing, it's another" is the sardonic title of an article by Li et al. ${ }^{1}$ which describes a puzzling inverse relationship between cancer and atherosclerosis. Observing that atherosclerosis is a major causative factor in cardiovascular disease, $\mathrm{Li}$ et al. identified that cardiovascular disease and cancer are the two leading causes of morbidity and mortality in developed nations. A recent 2019 analysis showed that cancer has

surpassed cardiovascular disease as the leading cause of death in high-income nations. ${ }^{2}$ While it may seem surprising that the associated risk of one of these diseases goes up as the other goes down, even more surprising is that no cause has been established to account for this inverse relationship, which has remained an unsolved mystery for decades. $\mathrm{Li}$ et al. noted that observations of the inverse association of cancer and atherosclerosis extend back as far as over half a century ago, but similar observations 
extend back even further than that. ${ }^{1}$ Elkeles $^{3}$ reviewed the research of early $20^{\text {th }}$ century pathologists, including Busch in 1924, Casper in 1932, Sjoeval and Wihman in 1934, and Wegelin in 1935, all of whom observed a very low frequency of advanced arteriosclerosis in cancer cases.

More recently, a prospective study that followed 5,262 elderly people for over 12 years found that deceased participants with symptomatic atherosclerotic disease, including coronary disease and atherosclerotic stroke, had approximately $30-40 \%$ reduced mortality from cancer compared to deceased participants without symptomatic atherosclerotic disease. ${ }^{4}$ Another analysis of 2,370 autopsy reports over 14 years found that cancers of the pancreas, breast, and colorectum, as well as lymphomas/lymphoid leukaemia and sarcomas had strong inverse correlations with atherosclerosis. ${ }^{5}$ One modern theory proposing a plausible explanation for the inverse association of cancer and atherosclerosis suggests that the administration of statins might increase the associated risk of cancer; however, a recent review of the literature suggested that statins were more likely associated with a decreased cancer risk. ${ }^{6}$ Furthermore, statins were obviously not in use during the early decades of the $20^{\text {th }}$ century when an inverse relationship between cancer and atherosclerosis was first observed. Another explanation for the inverse relationship of these two diseases proposed that chemotherapy lowered atherosclerosis in cancer patients, but Li et al. dismissed this proposal as unsubstantiated and inconsistent with the known pathobiology of atherosclerosis.'

$\mathrm{Li}$ et al. conducted an analysis of cancer and atherosclerosis based on 1,024 autopsy reports from Brigham and Women's Hospital in Cambridge, Massachusetts, USA, and the researchers conducted a second analysis from the database of the Harvard Catalyst Shared Health Research Information Network; both analyses confirmed a significant inverse relationship between cancer and atherosclerosis. ${ }^{1}$ The explanation offered by the researchers speculated upon a difference in patient propensity toward inflammatory properties, arguing that an inflammatory immune response appears more directly associated with atherosclerosis than with cancer. The researchers' hypotheses might help explain lower atherosclerosis among cancer cases, but it does not explain reports of lower cancer among atherosclerosis cases. Furthermore, the researchers' proposed explanations only account for a potential difference in disease mechanisms from a pathophysiological perspective, without considering a wider range of causes and factors associated with increased disease risks from an epidemiological perspective.

Shared modifiable risk factors, including smoking and poor nutrition, are associated with increased risks for both cardiovascular disease and cancer. ${ }^{7}$ Smoking tobacco is a risk factor associated with smoking-related cancers as well as atherosclerosis; however, there was a strong inverse association of nonsmoking related cancers and atherosclerosis in an analysis of 2,101 deceased patients. ${ }^{8}$ Aside from smoking, the current review article investigates poor nutrition as a risk factor for cancer and atherosclerosis, and proposes that different dietary patterns containing inverse ratios of phosphate and oxysterols, which are correspondingly associated with increased risks for cancer and atherosclerosis, may provide a novel hypothesis that explains the inverse associated risk of cancer and atherosclerosis.

\section{OXYSTEROLS AND ATHEROSCLEROSIS}

The following is a brief summary of the association of oxysterols and atherosclerosis, based on a more detailed review of the existing research literature. $^{9}$ Atherosclerosis is associated with serum levels of low-density lipoprotein cholesterol (LDL-C). ${ }^{10}$ As atherosclerosis develops, a lesion grows within the inner or intima layer of the arterial vascular wall." The inner layer of the vascular wall is lined with endothelial cells which form a barrier that normally regulates selective permeability of certain biomolecules from the blood plasma, but this permeability becomes dysregulated in cardiovascular disease. ${ }^{12}$ Endothelial cells are lined with membranes formed by a phospholipid bilayer which contains cholesterol molecules that strengthen the membrane. ${ }^{13}$

Oxysterols are cholesterol oxidation products that originate from endogenous and exogenous sources, including dietary sources of cholesterol that have undergone oxidation through processing, preparation, and storage. ${ }^{14}$ 
During the formation of oxysterols, oxidation changes the molecular structure and polarity of cholesterol, which creates packing defects in the vascular endothelial cell membranes as oxysterols enter into the phospholipid bilayer and do not line up properly compared to normal cholesterol. ${ }^{15}$ Packing defects from oxysterols eventually increase endothelial cell membrane permeability to protein from the blood plasma. ${ }^{16}$ This pathophysiological mechanism could explain unregulated permeability of serum LDL-C into the subendothelial space of the arterial wall, with subsequent LDL-C oxidation by immune cells and eventual formation of foam cells and an atheroma which extends into and blocks the lumen of the arterial vessel. ${ }^{17}$

In vitro experiments have demonstrated that oxysterols alter endothelial barrier permeability compared to normal cholesterol, ${ }^{18}$ and atherosclerosis in the aortas of rabbits increased dramatically when the rabbits were fed oxysterols. ${ }^{19}$ Although further research is needed to investigate the pathophysiological mechanism described above, clinical evidence continues to link oxysterols with atherosclerosis. For example, elevated serum levels of oxysterols in patients were associated with increased risk for atherosclerosis and cardiovascular disease..$^{20,21}$

Conventional dietary guidelines to reduce atherosclerosis, according to the Therapeutic Lifestyle Changes programme of the National Heart, Lung, and Blood Institute (NHLBI) in the USA, recommend reducing saturated fat to no more than $7 \%$ of calories and cholesterol to no more than $200 \mathrm{mg} /$ day. $^{22}$ More recently, the 2015 Dietary Guidelines Advisory Committee of the USA Department of Agriculture (USDA) and the U.S. Department of Health \& Human Services (HHS) issued a scientific report that is less restrictive of cholesterol intake. ${ }^{23}$ However, neither of these guidelines mention oxysterols, nor are oxysterols included in nutrient databases of foods.

Of relevance, symptoms of angina rapidly regressed in patients prescribed a vegan diet which contained no cholesterol, and presumably no oxysterols, but symptoms returned when dairy and eggs were added to the diet. ${ }^{24}$ In the first controlled clinical trial of a dietary intervention that reversed coronary heart disease, Ornish et al. ${ }^{25}$ used a vegetarian diet with small amounts of nonfat milk and egg whites, which contain little and no cholesterol, respectively. However, a more recent study found that stroke rates in vegetarians and vegans were $20 \%$ higher than in meat eaters, ${ }^{26}$ although the researchers could not identify causative dietary factors. Salt intake is associated with stroke in countries like China. ${ }^{27}$ Investigations should determine if vegans and vegetarians consume excessive salt in soy sauce, tamari, salted nuts, seeds, nut butters, processed snacks, exotic seasonings like Himalayan salt and sea salt, and high amounts of salt in baked grain products $^{28}$ and processed meat alternatives. ${ }^{29}$

A review examining the association between below-normal vitamin B12 status and cardiovascular health in vegans suggested that normal B12 levels might have a cardioprotective effect. ${ }^{30} \mathrm{~A}$ limitation of the reviewed studies is that changes observed in cardiovascular surrogates, flow-mediated endothelium-dependent dilation and carotid intima-media thickness, may not translate to actual cardiovascular events. Additionally, there are many vegan foods available that are fortified with vitamin B12, and the researchers suggested that vegans monitor their vitamin B12 status "to reap the full benefits of cardiovascular disease prevention in plantbased eating styles of vegan diets."30 Having reviewed evidence implicating dietary oxysterols in atherosclerosis, the next section of this article examines the leading cause of mortality in highincome nations: cancer, and its association with dysregulated dietary phosphate.

\section{PHOSPHATE TOXICITY AND TUMOURIGENESIS}

As risk factors for cancer increase through the global spread of Western lifestyles and an ageing population, cancer is projected to increase to 22.2 million new global cases in the year 2030. ${ }^{31}$ Substances that are identified as carcinogenic in laboratory analyses do not always progress to cancer in real life, ${ }^{32}$ implying that other cancer growth factors are involved in tumourigenesis. Schipper et al. ${ }^{33}$ suggested that cancer promotion is linked to dysregulated metabolic pathways which may be reversible. A recent review $^{34}$ supported the role of dysregulated dietary phosphate metabolism in the promotion and progression of tumourigenesis, which may be modified by a low-phosphate diet. 
Phosphate is formed from the essential micronutrient phosphorus, and inorganic phosphate in the body is normally regulated by endocrine communication between an axis of organs consisting of the kidneys, skeletal system, parathyroid glands, and intestines. ${ }^{35}$ If this axis becomes burdened, phosphate metabolism becomes dysregulated and extracellular and intracellular levels of phosphate may accumulate, leading to a harmful condition known as phosphate toxicity. Evidence suggests that excess phosphate may accumulate in the tumour microenvironment. For example, compared to normal cells, cancer cells from the ovaries, lung, breast, and thyroid overexpress sodiumphosphate cotransporters, ${ }^{36,37}$ which allow cells to absorb and sequester large amounts of inorganic phosphate from the tumour extracellular microenvironment. Tumour cells of the lung and colon were observed to contain levels of inorganic phosphate that were up to twice as high as normal cells. ${ }^{38}$ Excess inorganic phosphate was found to increase biogenesis of ribosomal RNA, which stimulates protein synthesis and promotes cancer cell growth. ${ }^{39}$ Using animal models, researchers found that high levels of dietary phosphate increased growth of skin cancer ${ }^{40}$ and lung cancer, ${ }^{41}$ activating cell-signalling pathways involving PI3K, protein kinase $\mathrm{B}$, and $\mathrm{mTOR}^{42}$ Progression of cancer in metastasis has also been linked to high concentrations of phosphate in extracellular tissue. ${ }^{43}$

Higher serum phosphate levels were positively associated with cancer in adults, ${ }^{44,45}$ except in females with reproductive cancers, possibly related to a shift of high serum phosphate levels into rapidly growing reproductive tissue. Of relevance to the present article, a ketogenic diet reduced tumours in experimental animals ${ }^{46}$ and in patients with brain cancer. ${ }^{47}$ A sample ketogenic diet used to medically treat children with epilepsy provides a 4:1 ratio of fat $g$ to nonfat 9.48 Extrapolated to a diet sufficient in calories for an adult, the low-phosphate level of the ketogenic diet lies below the recommended dietary intake of $700 \mathrm{mg}$ of phosphorus per day for an adult. Nevertheless, despite benefits associated with reducing cancer risk, the high-fat ketogenic diet has also been associated with arterial wall dysfunction in children and adults with epilepsy. ${ }^{49}$

\section{ATHEROGENIC AND TUMOURIGENIC DIETARY PATTERNS}

The preceding literature review provides evidence that an atherogenic dietary pattern is high in cholesterol, saturated fat, and oxysterols, and a tumourigenic dietary pattern is high in phosphate as well as protein. Dietary phosphate is closely correlated with dietary protein, with approximately 12-14 mg phosphorus for each $\mathrm{g}$ of protein..$^{50}$ Therefore, as the macronutrient ratio of protein increases in a dietary pattern, phosphate often also increases.

Figure 1 compares a proposed inverse ratio of phosphate and oxysterols in atherogenic and tumourigenic dietary patterns. Note that as the public is encouraged to consume more low-fat and nonfat foods, which reduce the associated risk of atherosclerosis, the macronutrient proportion of proteins and phosphate increases in the diet, thereby increasing the associated risk of cancer linked to high phosphate intake. Inversely, as the public consumes a greater proportion of animalbased foods high in saturated fat, cholesterol, and oxysterols, the associated risk of atherosclerosis increases while the lower macronutrient proportion of protein and phosphate in the diet reduces the associated risk of cancer.

\section{FUTURE PREVENTION OF CANCER AND ATHEROSCLEROSIS}

An optimal solution to the dilemma of an inverse association between atherosclerosis and cancer might be to lower dietary intake of both phosphate and oxysterols by increasing dietary intake of fruits, vegetables, and plant-based fats, as in a Mediterranean dietary pattern. ${ }^{51} \mathrm{~A}$ plant-based dietary pattern is associated with lower cardiovascular disease risk and mortality in middle-aged adults. ${ }^{52}$ Dietary guidelines for cancer prevention from the American Cancer Society (ACS) ${ }^{53}$ and the World Cancer Research Fund/American Institute for Cancer Research (WCRF/AICR) $)^{54}$ also emphasise a wholefoods plant-based dietary pattern with limited consumption of refined grain products and processed meat. In addition, the ACS guidelines provide advice on properly balancing strict vegetarian or vegan diets with vitamin B12, zinc, iron, and calcium to meet the special needs of premenopausal women and children. 


\section{Inverse ratio of phosphate and oxysterols}

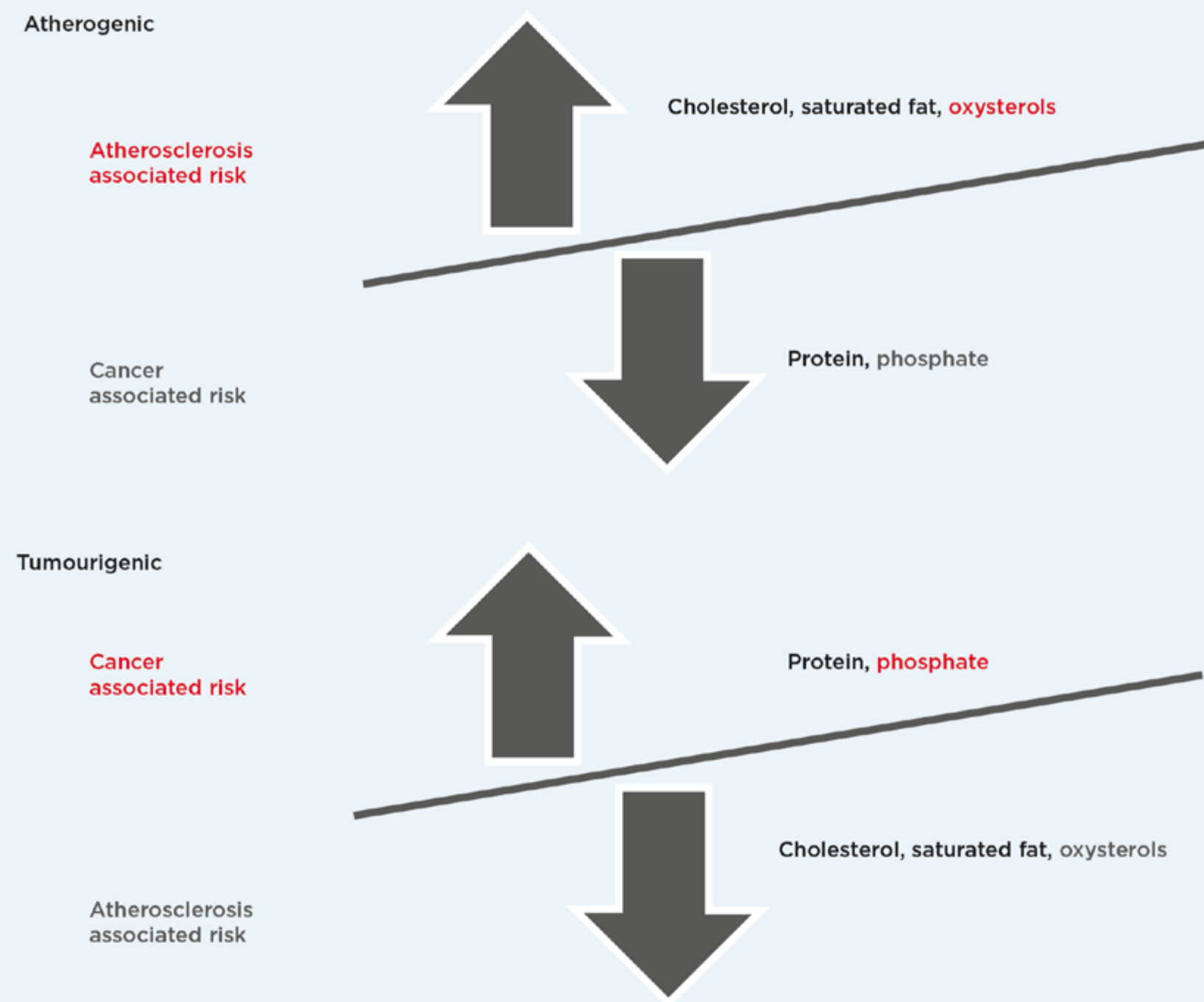

Figure 1: Dietary patterns.

Proposed inverse ratio of phosphate and oxysterols in atherogenic and tumourigenic dietary patterns.

Specific cancer sites associated with different dietary factors are also mentioned in the ACS guidelines, such as cancers of the breast, colorectum, endometrium, and other organs. Tumourigenic properties of excess phosphate is an overlooked dietary factor that may be involved in many of these specific cancers, and more research is needed to investigate the effects of excess phosphate by specific cancer site. In addition, many specific food items such as full-fat meats, dairy, and eggs have high levels of animal protein and phosphorus in addition to high levels of fat and cholesterol, thus increasing the associated risk of both cardiovascular disease and cancer in investigations that include these foods.
Nevertheless, the overall dietary pattern, not any particular food, could be the determining factor in the inverse association between atherogenic and tumourigenic diets.

Table $1^{55}$ shows the low phosphorus content of selected fruit and vegetables compared to grains, legumes, and animal-based foods. ${ }^{55} \mathrm{~A}$ recent meta-analysis of 95 studies found that intake of fruit and vegetables was associated with significant reductions in cardiovascular disease and cancer. ${ }^{56}$ The researchers observed dose-response reductions in diseases associated with daily combined fresh fruit and vegetable intakes of up to $800 \mathrm{~g}$ for cardiovascular disease and $600 \mathrm{~g}$ for cancer. 
Table 1: Phosphorus in selected food items.

\begin{tabular}{|l|l|l|l|}
\hline Food item & Phosphorus $\mathbf{( m g / 1 0 0 ~ g ) ~}$ & Food item & Phosphorus (mg/100 g) \\
\hline Pineapple & 8 & Potato & 57 \\
\hline Grapefruit & 8 & Date & 62 \\
\hline Apple & 11 & Broccoli & 67 \\
\hline Pear & 12 & Whole milk & 84 \\
\hline Fig & 14 & Green peas & 108 \\
\hline Orange & 14 & Wholegrain pasta & 110 \\
\hline Cantaloupe & 15 & Tilapia & 170 \\
\hline Grapes & 20 & Chicken breast & 174 \\
\hline Banana & 22 & Whole egg & 198 \\
\hline Celery & 24 & Sirloin & 209 \\
\hline Tomato & Chickpeas & 252 \\
\hline Carrot & 24 & Lentils & 281 \\
\hline Romaine & 35 & Black beans & 352 \\
\hline Kale & 35 & Cheddar cheese & 455 \\
\hline
\end{tabular}

Adapted from U.S. Department of Agriculture, Agricultural Research Service. ${ }^{55}$

This amount of fruit and vegetables is double the $400 \mathrm{~g}$ currently recommended by the World Health Organization $(\mathrm{WHO})^{57}$ and the World Cancer Research Fund (WCRF). ${ }^{58}$

Plant-based dietary fats, like olive oil in the Mediterranean diet, have also been associated with lower cancer and atherosclerosis risk. ${ }^{59}$ Of relevance, oils obtained from plant-based foods are generally stripped of the mineral content found in whole foods, so these oils often contain little or no phosphorus. In addition, cholesterol is lacking in plant-based foods, providing little chance for the formation of cholesterol oxidation products in plant-based oils. Whole foods such as nuts, seeds, coconuts, and avocados contain moderate to high amounts of phosphorus. But because these plant-based foods are also high in fat and calories, their overall phosphorus caloric density, or phosphorus per calorie, is relatively low, especially compared to the very high phosphorus caloric density of lean flesh foods, nonfat dairy, legumes, and grain products.

\section{CONCLUSION}

This article proposes a novel hypothesis suggesting that the answer to the nearly centuryold riddle of an inverse relationship between atherosclerosis and cancer may be explained by inverse proportions of phosphate and oxysterols in atherogenic and tumourigenic dietary patterns. Furthermore, evidence linking reduced associated risks for cancer and cardiovascular disease with plant-based dietary patterns, especially diets abundant in fruits and vegetables with moderate amounts of plant-based fats, infers that a substantial change in current dietary patterns of developed nations is necessary for the future prevention of cardiovascular disease and cancer. 


\section{References}

1. Li M et al. If it's not one

thing, it's another: an inverse relationship of malignancy and atherosclerotic disease. PLoS One. 2015;10(5):e0126855.

2. Dagenais $\mathrm{G}$ et al. Variations in common diseases, hospital admissions, and deaths in middleaged adults in 21 countries from five continents (PURE): a prospective cohort study. Erratum in: Lancet. 2020;395(10226):785-94

3. Elkeles A. Cancer and atherosclerosis. $\mathrm{Br} J$ Cancer. 1956;10(2):247-50

4. Benito-Leon J et al. Symptomatic atherosclerotic disease and decreased risk of cancer-specific mortality: a prospective, populationbased study (NEDICES). Medicine. 2015:94(32):e1287.

5. Jirasek $A$ et al. Evaluation of general and coronary atherosclerosis and malignant disease demonstrates inverse correlations for specific cancer types as well as cancer in general. Pathol Res Pract. 2016;212(11):988-94

6. Mistiaen W. Statins and malignancies in cardiovascular practice. Int J Cardiol Lipidol Res. 2017;4(2):16-21.

7. Koene $\mathrm{R}$ et al. Shared risk factors in cardiovascular disease and cancer. Circulation. 2016;133(11):1104-14.

8. Budczies $\mathrm{J}$ et al. Comprehensive analysis of clinico-pathological data reveals heterogeneous relations between atherosclerosis and cancer. Clin Pathol. 2014;67(6):482-90

9. Brown R. Phospholipid packing defects and oxysterols in atherosclerosis: dietary prevention and the French paradox. Biochimie. 2019;167:145-51.

10. Linton $M$ et al. "The Role of Lipids and Lipoproteins in Atherosclerosis," Feingold KR et al. (eds.), Endotext [Internet] (2000), South Dartmouth: MDText.com, Inc.

11. Glagov S et al. Compensatory enlargement of human atherosclerotic coronary arteries. NEJM. 1987;316(22):1371-5.

12. Yuan S, Rigor R, "Regulation of Endothelial Barrier Function," Granger D, Granger J (eds.), Colloquium Series on Integrated Systems Physiology: From Molecule to Function (2011), San Rafael: Morgan \& Claypool Publishers.

13. Alberts B et al. "The Lipid Bilayer," Molecular Biology of the Cell (2002), $4^{\text {th }}$ edition, New York City: Garland Science.

14. Kulig W et al. Cholesterol oxidation products and their biological importance. Chem Phys Lipids. 2016:199:144-60.
15. Olkkonen V, Hynynen R. Interactions of oxysterols with membranes and proteins. Molecular aspects of medicine. 2009;30(3):123-33.

16. Pinot $\mathrm{M}$ et al. Feedback between membrane tension, lipid shape and curvature in the formation of packing defects. bioRxiv. 2018:389627.

17. Zhang $X$ et al. Endothelial transcytosis of lipoproteins in atherosclerosis. Front Cardiovasc Med. 2018;5:130.

18. Kulig WT et al. Oxidation of cholesterol changes the permeability of lipid membranes. Biophysical Journal. 2017;112(3):377a.

19. Staprans I et al. The role of dietary oxidized cholesterol and oxidized fatty acids in the development of atherosclerosis. Mol Nutr Food Res. 2005;49(11):1075-82.

20. Pordal A-H et al. Plasma oxysterol level in patients with coronary artery stenosis and its changes in response to the treatment with atorvastatin. MJIRI. 2015;29:192

21. Song $\mathrm{J}$ et al. Association of plasma 7-ketocholesterol with cardiovascular outcomes and total mortality in patients with coronary artery disease. Circ Res. 2017;120(10):1622-31.

22. National Institutes of Health: Nationa Heart, Lung and Blood Institute. Your guide to lowering your cholesterol with TLC: therapeutic lifestyle changes. 2005. Available at: https:// www.nhlbi.nih.gov/files/docs/public/ heart/chol_tlc.pdf.

23. United States Department of Agriculture. Scientific report of the 2015 Dietary Guidelines Advisory Committee: advisory report to the Secretary of Health and Human Services and the Secretary of Agriculture. 2015. Available at: https://health.gov/sites/default/ files/2019-09/Scientific-Reportof-the-2015-Dietary-GuidelinesAdvisory-Committee.pdf

24. Ellis F, Sanders T. Angina and vegan diet. Am Heart J. 1977;93(6):803-5.

25. Ornish $D$ et al. Can lifestyle changes reverse coronary heart disease? The lifestyle heart trial. Lancet. 1990;336(8708):129-33.

26. Tong et al. Risks of ischaemic heart disease and stroke in meat eaters, fish eaters, and vegetarians over 18 years of follow-up: results from the prospective EPIC-Oxford study. BMJ. 2019;366:14897.

27. Li $Y$ et al. Longitudinal change of perceived salt intake and stroke risk in a Chinese population. Stroke. 2018;49(6):1332-9.

28. Stall S. Bread has salt: options for very low-sodium bread. Journal of Renal Nutrition. 2013;23(1):e5-e9.
29. actiononsalt.org.uk. Meat Alternatives Survey 2018: Action on salt expose excessive amounts of salt in 'healthy' processed meat alternatives and demand urgent action from Public Health England [Available from: http://www.actiononsalt.org.uk/ salt-surveys/2018/meat-alternativessurvey/.

30. Woo K et al. Vegan diet, subnormal vitamin B-12 status and cardiovascular health. Nutrients. 2014;6(8):3259-73.

31. Bray F et al. Global cancer transitions according to the Human Development Index (2008-2030): a population-based study. Lancet Oncol. 2012;13(8):790-801.

32. cancer.org. Known and Probable Human Carcinogens, American Cancer Society. 2020 [Available from: https:// www.cancer.org/cancer/cancercauses/general-info/known-andprobable-human-carcinogens.html.

33. Schipper $\mathrm{H}$ et al. A new biological framework for cancer research. Lancet. 1996;348(9035):1149-51.

34. Brown R, Razzaque M. Phosphate toxicity and tumorigenesis. Biochim Biophys Acta Rev Cancer. 2018;1869(2):303-309.

35. Brown R, Razzaque M. Dysregulation of phosphate metabolism and conditions associated with phosphate toxicity. Bonekey Rep. 2015;4:705.

36. Levan $\mathrm{K}$ et al. Immunohistochemical evaluation of epithelial ovarian carcinomas identifies three different expression patterns of the MX35 antigen, $\mathrm{NaPi} 2 \mathrm{~b}$. BMC cancer 2017:17(1):303.

37. D'Arcangelo M et al. 194P prevalence and prognostic significance of sodium-dependent phosphate transporter 2B (NAPI2B) protein expression in non-small cell lung cancer (NSCLC). Annals of Oncology. 2014;25(suppl_4):iv66-iv7.

38. Elser $\mathrm{J}$ et al. Biological stoichiometry in human cancer. Plos one. 2007;2(10):e1028.

39. Ward D, Griffin A. Phosphorus incorporation into nucleic acids and proteins of liver nuclei of normal and azo dye-fed rats. Cancer Res. 1955;15(7):456-61.

40. Camalier $\mathrm{C}$ et al. Elevated phosphate activates $\mathrm{N}$-ras and promotes cell transformation and skin tumorigenesis. Cancer Prev Res (Phila). 2010;3(3):359-70

41. Jin $\mathrm{H}$ et al. High dietary inorganic phosphate increases lung tumorigenesis and alters Akt signaling. Am J Respir Crit Care Med. 2009;179(1):59-68.

42. Guertin D, Sabatini D. Defining the role of $\mathrm{mTOR}$ in cancer. Cancer Cell. 2007;12(1):9-22. 
43. Bobko A et al. Interstitial inorganic phosphate as a tumor microenvironment marker for tumor progression. Sci Rep. 2017;7:41233.

44. Papaloucas $C$ et al. Measurement of blood phosphorus: a quick and inexpensive method for detection of the existence of cancer in the body. Too good to be true, or forgotten knowledge of the past? Med Hypotheses. 2014;82(1):24-5.

45. Wulaningsih $\mathbf{W}$ et al. Inorganic phosphate and the risk of cancer in the Swedish AMORIS study. BMC Cancer. 2013;13(1):257.

46. Varshneya $\mathrm{K}$ et al. The efficacy of ketogenic diet and associated hypoglycemia as an adjuvant therapy for high-grade gliomas: a review of the literature. Cureus. 2015;7(2):e251.

47. Schwartz $\mathrm{K}$ et al. Treatment of glioma patients with ketogenic diets: report of two cases treated with an IRBapproved energy-restricted ketogenic diet protocol and review of the literature. Cancer Metab. 2015;3(1):3.

48. Lee $\mathrm{E}$ et al. Ketogenic diet for children with epilepsy: a practical meal plan in a hospital. Clin Nutr Res. 2016;5(1):60-3.

49. Coppola $\mathrm{G}$ et al. The impact of the ketogenic diet on arterial morphology and endothelial function in children and young adults with epilepsy: a case-control study. Seizure. 2014;23(4):260-5

50. D'Alessandro $C$ et al. The "phosphorus pyramid": a visual tool for dietary phosphate management in dialysis and CKD patients. BMC Nephrol. 2015;16:9.

51. Boucher JL. Mediterranean eating pattern. Diabetes Spectr. 2017;30(2):72-6

52. Kim $\mathrm{H}$ et al. Plant-based diets are associated with a lower risk of incident cardiovascular disease, cardiovascular disease mortality, and all-cause mortality in a general population of middle-aged adults. J Am Heart Assoc. 2019;8(16):e012865.

53. Kushi LH et al; American Cancer Society 2010 Nutrition and Physical Activity Guidelines Advisory Committee. American Cancer Society Guidelines on nutrition and physical activity for cancer prevention: reducing the risk of cancer with healthy food choices and physical activity. CA Cancer J Clin. 2012:62(1):30-67.

54. World Cancer Research Fund/ American Institute for Cancer Research. Diet, nutrition, physical activity and cancer: a global perspective. Continuous Update Project Expert Report 2018.

55. U.S. Department of Agriculture Agricultural Research Service. FoodData Central, 2019. Available at: https://fdc.nal.usda.gov.

56. Aune D et al. Fruit and vegetable intake and the risk of cardiovascular disease, total cancer and all-cause mortality-a systematic review and dose-response meta-analysis of prospective studies. Int J Epidemiol. 2017;46(3):1029-56.

57. World Health Organization. Promoting fruit and vegetable consumption around the world. Global Strategy on Diet, Physical Activity and Health; World Health Organization: Geneva, Switzerland. 2003. Available at: https://www.who. int/dietphysicalactivity/fruit/en/

58. Clinton S et al. The World Cancer Research Fund/American Institute for Cancer Research third expert report on diet, nutrition, physical activity, and cancer: impact and future directions. J Nutr. 2020;150(4):663-71.

59. Mentella M et al. Cancer and mediterranean diet: a review. Nutrients. 2019;11(9):2059. 